УДК 669.017.3:536.421.4

DOI: $10.17308 / \mathrm{kcmf} .2019 .21 / 711$

Поступила в редакцию 30.11 .2018

Подписана в печать 15.02.2018

\title{
МОДЕЛЬ МИКРОКРИСТАЛЛИЗАЦИИ \\ 50 \% ДВУХКОМПОНЕНТНЫХ МЕТАЛЛИЧЕСКИХ РАСПЛАВОВ В ДИФФУЗИОННО-РЕЛАКСАЦИОННОМ РЕЖИМЕ
}

\author{
() 2019 Ю. А. Байков ${ }^{1}$ Н. И. Петров ${ }^{* 1}$, М. И. Тимошина ${ }^{2}$, Е. В. Акимов ${ }^{2}$ \\ ${ }^{1}$ Российский химико-технологический университет имени Д. И. Менделеева \\ Миусская пл., 9, 125047 Москва, Российская Федераџия \\ ${ }^{2}$ Московский технический университет связи и информатики \\ Авиамоторная ул., 8a, 111024, Москва, Российская Федераџия
}

\begin{abstract}
Аннотация. Рассмотрена модель микрокристаллизации 50 \% двухкомпонентных металлических расплавов в диффузионно-релаксационном режиме. При этом развита модель переходной двухфазной зоны (ПДЗ) в пространстве концентраций мономеров роста, принадлежащих двум агрегатным состояниям - расплаву и кристаллу. Записаны кинетические дифференциальноразностные уравнения, описывающие эволюцию структуры ПДЗ во времени с учетом ее «ступенчатой» формы, образованной механизмом спонтанных флуктуаций с ограниченным спектром изменения концентраций мономеров роста (в модели ПДЗ) во всех монослоях переходной конечной области, отделяющей собой двухкомпонентный расплав от кристаллической фазы. Учтены зависимости частот обмена мономерами роста между расплавом и кристаллом от энергий связи двух ближайших мономеров и от температуры кристаллизующейся системы расплав-кристалл. Эта модель соответствует схеме реальной конечной протяженности поверхности раздела двух соприкасающихся фаз и носит название кристалла Косселя-Странского.

Ключевые слова: переходная двухфазная зона, мономеры роста, параметр дальнего порядка, высоты изломов, образованных концентрациями мономеров роста кристаллической фазы, параметр «шероховатости» ПДЗ, скорость кристаллизации.
\end{abstract}

\section{ВВЕДЕНИЕ}

При создании современных металлических материалов, отвечающих требованиям практического их использования, весьма насущным является изучение физической природы фазовых переходов при образовании кристаллических систем в зависимости от выбранной модели их микрокристаллизации. Весьма актуально развитие теории фазовых переходов или специфических физических эффектов в концентрированных кристаллических фазах, формирующихся из различных исходных маточных сред. К ним относится теория кристаллизации. Весьма актуальным является изучение различия в кинетике кристаллизации металлов и сплавов с одной стороны и неметаллами с другой. В частности, при кристаллизации одно- и многокомпонентных металлических расплавов сущест-

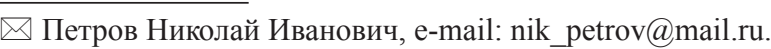

венную роль должна играть морфология поверхности раздела двух соприкасающихся фаз системы расплав-кристалл в двух известных в кристаллографии режимах - кинетическом (бездиффузионном) и диффузионно-релаксационном. Эти различия предполагается описать и изучить в пределах некоторой переходной области - переходной двухфазной зоны (ПДЗ), отделяющей две соприкасающиеся массивные фазы в двух вышеупомянутых режимах. В кинетическом режиме эта ПДЗ подробно изучена в работах [1-7].

В этих работах в целях теоретического удобства описания морфологии переходной двухфазной зоны, отделяющей собой металлический расплав от кристалла, были введены понятия мономеров роста в пространстве концентраций двух возможных агрегатных состояний (расплав и кристалл), заменяющих собой реальные металлические мик- 
рочастицы в реальных переходных межфазных областях. В работах [1-7] используемая модель переходной двухфазной зоны (ПДЗ) в пространстве концентраций мономеров роста имела «ступенчатую» форму (см. рис. 1), обусловленную действием механизма спонтанных флуктуаций с ограниченным спектром изменения концентраций мономеров роста твердой фазы. Такая схема ПДЗ имела сходство с известными свойствами реальных поверхностей раздела системы расплав-кристалл, а именно: ее конечность $-n_{0}$ (число моноатомной толщины слоев ПДЗ) и ступень с изломами. Эти реальные поверхности раздела носят название поверхности Косселя-Странского.

По оси абсцисс рис. 1 отложены номера моноатомной толщины слоев ПДЗ, отделяющих собой кристалл от двухкомпонентного металлического расплава. По оси ординат отложены величины концентраций мономеров роста, находящихся в кристаллическом состоянии (заштрихованные столбики $c_{i}=\frac{m_{i}}{N}$, где $m_{i}-$ число мономеров роста кристалла в слое $i, N$ - общее количество мономеров роста обоих состояний (жидкого и кристалличес- кого и обоих сортов) в каждом слое ПДЗ толщины $d$, характеризующей размер одного мономера роста вдоль каждого из трех направлений объемной переходной двухфазной зоны. Число $N=$ const. На рис. 1 представлено сечение ПДЗ плоскостью $Z=0$ (плоскостью рисунка) [8]. Незаштрихованные части каждого из $n_{0}$ столбиков толщины $d$ определяют концентрации мономеров роста, находящихся в двухкомпонентном 50 \% металлическом расплаве. Каждый заштрихованный столбик на рис. 1 в случае двухкомпонентной системы расплав-кристалл предполагается состоящим из конечного числа мономеров, обозначаемых как $\alpha N^{-1}, \beta N^{-1}, \gamma N^{-1}$ и т. д., где $\alpha, \beta, \gamma=A, B$ - индексы, отмечающие сортность того или иного мономера роста, каждый из которых находится в кристаллическом состоянии. Всего мономеров роста кристаллического состояния во всех $n_{0}$-монослоях ПДЗ очевидно есть $N \sum_{i=1}^{n_{0}} \bar{c}_{i}[9,10]$. Соответственно число мономеров роста жидкого состояния во всех $n_{0}$ слоях ПДЗ есть $N\left(n_{0}-\sum_{i=1}^{n_{0}} \bar{c}_{i}\right)$. Фигурирующие здесь концентрации $\bar{c}_{i}=\left\langle c_{i}\right\rangle$ являются ус-

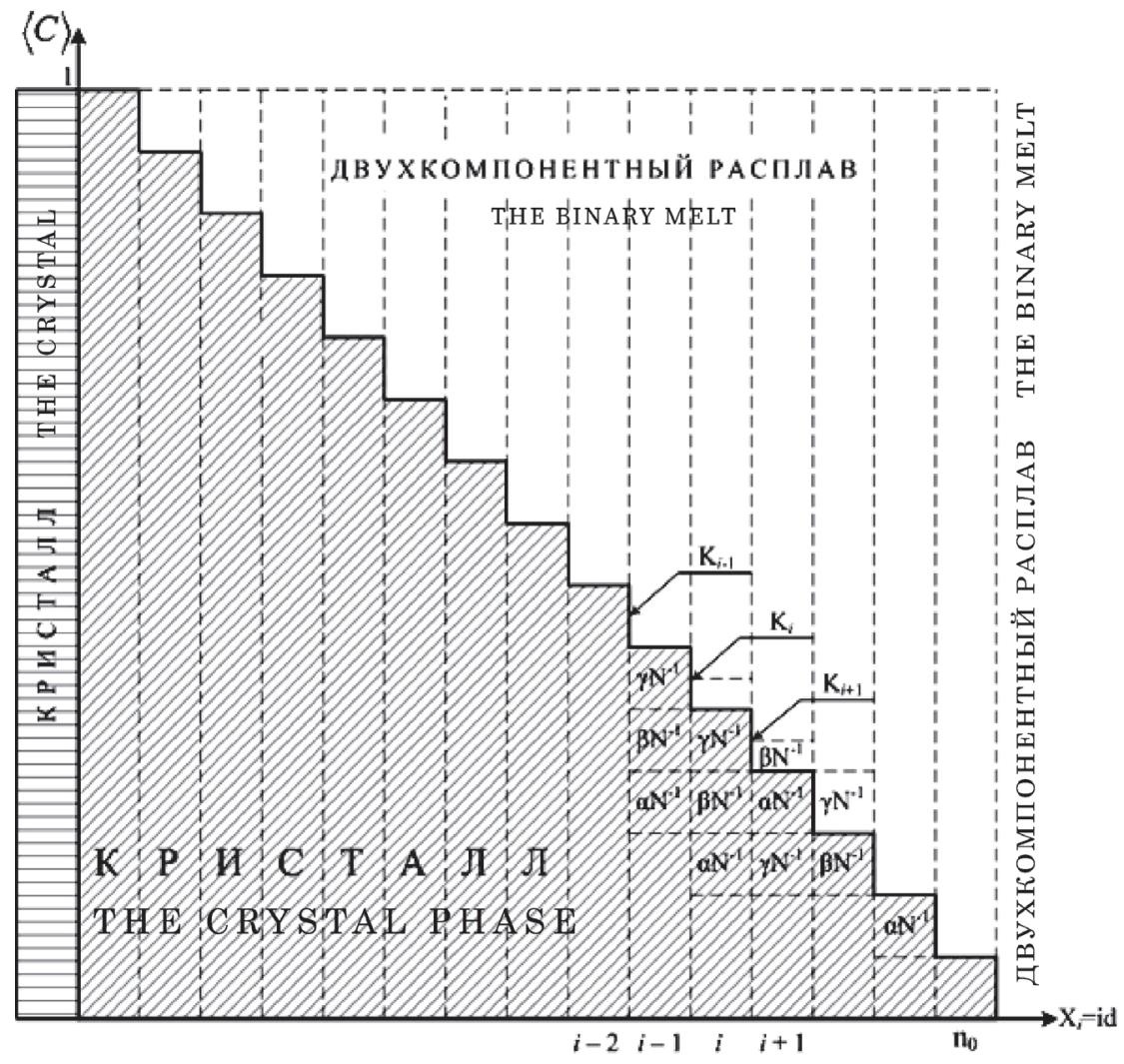

Рис. 1. Модель переходной двухфазной зоны (ПДЗ) «ступенчатой» формы в пространстве концентраций мономеров роста

[Fig. 1. The step-like transitional diphase zone (DTZ) model presented in the growth monomers concentration space] 
редненными величинами в соответствии с функциями распределения вероятностей реализации спонтанных флуктуаций концентраций частиц твердого состояния (мономеров роста в модели ПДЗ) с ограниченным спектром их изменения (см. [5, 11, $12,13])$. Конечной протяженности структура ПДЗ $\left(n_{0}=\right.$ const $)$ характеризуется величинами изломов границы раздела двух соприкасающихся двухкомпонентных фаз (расплав-кристалл). В процессе действия флуктуационного механизма с ограниченным спектром в слоях ПДЗ оказывается, что в результате флуктуации концентрации мономеров роста твердой фазы в $i$-слое $\left(i=1,2, \ldots, n_{0}\right)$ данная концентрация не может быть больше соответствующей концентрации мономеров роста твердого состояния в соседнем $(i-1)$ слое и не может быть меньше аналогичной концентрации в соседнем $(i+1)$ слое ПДЗ. В результате величины (высоты) этих изломов, определяемые как $K_{i-1}=\left\langle C_{i-1}\right\rangle-\left\langle C_{i-2}\right\rangle$, $K_{i}=\left\langle C_{i}\right\rangle-\left\langle C_{i-1}\right\rangle, K_{i+1}=\left\langle C_{i+1}\right\rangle-\left\langle C_{i}\right\rangle$, оказываются неположительными при всех $i=1, \ldots, n_{0}-1$. На рис. 1 высоты этих изломов показаны на границах монослоев $(i-2),(i-1) ;(i-1), i$ и $i,(i+1)$. В направлении оси $Z$, направленной вертикально вниз по отношению к плоскости указанного сечения (плоскость рисунка), за каждым из отмеченных мономеров роста, находящихся в определенном агрегатном состоянии, находится некий мономер роста того же агрегатного состояния. Выше плоскости сечения в модели ПДЗ какие-либо мономеры роста, по определению, отсутствуют.

\section{ПОСТАНОВКА ЗАДАЧИ}

На первой стадии, используя понятия мономеров роста двух агрегатных состояний (двухкомпонентные расплав и кристалл) и связанную с ними модель переходной двухфазной зоны (ПДЗ) в пространстве концентраций вышеупомянутых мономеров роста, необходимо описать структуру и эволюцию данной ПДЗ в условиях диффузионно-релаксационного режима кристаллизации 50 \% двухкомпонентного металлического расплава. При этом необходимо учесть зависимости частот обмена мономерами роста в модели ПДЗ в процессе ее эволюции от энергий взаимодействия данных мономеров разных агрегатных состояний и от температуры (переохлаждения) кристаллизующейся системы расплав-кристалл. При реализации диффузионно-релаксационного режима кристаллизации 50 \% двухкомпонентных металлических расплавов необходимо учесть зависимость от времени всех основных характеристических функций, описываю- щих структуру ПДЗ, а также введение времен релаксации, фигурирующих в исходных дифференциально-разностных уравнениях описания эволюции ПДЗ. Средняя скорость кристаллизации 50 \% двухкомпонентных металлических расплавов в модели ПДЗ должна иметь зависимость от времени.

На второй стадии исследования ставится задача отыскания критических температур (переохлаждений системы расплав-кристалл), при достижении которых хаос двухкомпонентного расплава передается двухкомпонентной кристаллической фазе стехиометрического состава с простой кубической ячейкой, а также нахождение времен релаксации, по прошествии которых восстанавливается упорядоченная структура двухкомпонентного кристалла (в понятиях параметров дальнего порядка). При этом ставятся задачи в условиях диффузионно-релаксационного режима кристаллизации найти математические законы, описывающие как процесс разупорядочения двухкомпонентной кристаллической фазы, так и обратный процесс восстановления со временем регулярного упорядоченного двухкомпонентного кристалла с элементарной кубической решеткой и стехиометрического состава.

Одновременно ставится задача определения особенностей в кинетике эволюции переходной двухфазной зоны (ПДЗ) в условиях диффузионнорелаксационного режима при образовании полностью разупорядоченной и упорядоченной двухкомпонентной кристаллической фазы.

\section{СТРУКТУРА ПЕРЕХОДНОЙ ДВУХФАЗНОЙ ЗОНЫ}

Сама структура ПДЗ описывается наборами тех же функций, что были использованы в работах [5-13], но в новом диффузионно-релаксационном режиме кристаллизации двухкомпонентных 50 \% металлических расплавов при образовании кристаллической двухкомпонентной фазы с простой кубической элементарной решеткой (кубическая сингония) и стехиометрического состава. Это означает, что функции, описывающие структуру ПДЗ, зависят от времени. Таким образом, структура переходной двухфазной зоны, не меняя своей кристаллографической симметрии - протяженности и кубической сингонии, перераспределяется внутри себя по составу мономеров роста, занимающих узлы двух подрешеток кристаллической фазы, на которые разбивается исходная кубическая простая ячейка. Физически это означает, что первоначально полностью, возможно, разупорядоченная двухкомпонентная кристаллическая фаза в данном режиме 
кристаллизации с течением времени получает возможность перейти в полностью упорядоченное состояние. При этом для систем с элементарной простой кубической ячейкой и стехиометрического состава, параметр дальнего порядка $\eta(t)$, определяемый аналогично работам [5-13], является функцией времени и может изменяться в пределах $0 \leq \eta(t) \leq 1$. Это свойство параметра $\eta(t)$ изменяться с течением времени в указанных приделах сохраняется при любых допустимых переохлаждениях системы двухкомпонентный расплав-кристалл, что было невозможно при кинетическом режиме кристаллизации двухкомпонентных 50 \% металлических расплавов (см. [1-4]). Очевидно, будет иметь место некий релаксационный процесс, характеризуемый временем релаксации и коэффициентом диффузии.

Для систем с простой кубической ячейкой и стехиометрического состава, когда данная простая кубическая решетка разбивается на две эквивалентные подрешетки с чередующимися узлами, вводят функции $X_{\alpha}^{(i)}$, определяющие априорную вероятность замещения мономерами роста сорта « $\alpha »$ какого-либо узла, принадлежащего $i$-ой подрешетке $(\alpha=A, B ; i=1,2)$. Эти вероятности зависят от параметра дальнего порядка $\eta(t)$ и имеют вид:

$$
\begin{aligned}
& X_{A}^{(1)}(t)=\frac{1}{2}(1+\eta(t)), X_{B}^{(1)}(t)=\frac{1}{2}(1-\eta(t)), \\
& X_{A}^{(2)}(t)=\frac{1}{2}(1-\eta(t)), X_{B}^{(2)}(t)=\frac{1}{2}(1+\eta(t))
\end{aligned}
$$

Если ввести числа $\bar{N}, \bar{N}^{(1)}, \bar{N}^{(2)}$, обозначающие общее количество узлов твердой фазы обеих подрешеток, подрешеток первого и второго типов соответственно, то параметр дальнего $\eta(t)$ порядка можно определить следующим образом

$$
\eta(t)=\frac{X_{A}^{(1)}(t)-v_{0}}{1-v_{0}}
$$

где $\vee_{0}=\frac{\bar{N}^{(1)}}{\bar{N}}, 1-\mathrm{v}_{0}=\frac{\bar{N}^{(2)}}{\bar{N}}$.

При этом полностью разупорядоченная двухкомпонентная кристаллическая структура с простой кубической ячейкой и стехиометрического состава характеризуется следующими величинами априорных вероятностей:

$$
X_{A}^{(1)}=X_{A}^{(2)}=v_{0}=C_{A}, X_{B}^{(1)}=X_{B}^{(2)}=1-v_{0}=C_{B}
$$

где $C_{A}$ и $C_{B}-$ концентрации мономеров роста в кристаллической фазе в каждом заштрихованном столбике ПДЗ соответственно сортов $A$ и $B$

Функции, определяющие структуру всех $n_{0}$ монослоев переходной двухфазной зоны в пространстве концентраций мономеров роста кристалли- ческой фазы (см. рис. 1) в произвольный момент времени « $t$ » по аналогии с подобными функциями в кинетическом режиме следующие. Пусть функция $\mathrm{X}_{\alpha \beta}^{(j k)}\left(K_{i}, K_{i+1}, t\right)$ есть вероятность обнаружить столбцы кристаллической фазы в модели ПДЗ, оканчивающиеся сверху мономерами роста $\alpha N^{-1}$, $\beta N^{-1}$, принадлежащими узлам подрешеток « $i »$ и «k» соответственно, в конфигурации с изломами $K_{i}$ и $K_{i+1}$ слева и справа от него в момент времени «t» $\left(\alpha, \beta=A, B ; j, k=1,2 ; i=1,2, \ldots n_{0}\right)$. Функция $\mathrm{X}_{\alpha \beta}^{(j k)}\left(K_{i}, t\right)$ есть вероятность обнаружить столбик, оканчивающийся мономерами роста $\alpha N^{-1}$, $\beta N^{-1}$,принадлежащими соответственно узлам подрешеток « $j »$ и « $k »$ у излома высоты $K_{i}$ в тот же момент времени «t» $\left(i=1,2, \ldots, n_{0}\right)$. Далее, например, функция $\mathrm{X}_{\alpha \beta \gamma}^{(j k j)}\left(K_{i}+N^{-1}, K_{i+1}, t\right)$ есть вероятность обнаружить столбик, оканчивающийся мономерами роста $\alpha N^{-1}, \beta N^{-1}, \gamma N^{-1}$, принадлежащими узлам подрешеток $j, k, j$ соответственно, в конфигурации с изломами $K_{i}+N^{-1}$ слева и $K_{i+1}$ справа от него в момент времени «t» $\left(i=1,2, \ldots, n_{0}\right)$. Введение подобных функций совершенно необходимо для описания эволюции кристаллизующейся ПДЗ во времени с меняющейся структурой.

Обмен мономерами роста разных сортов и в разных структурных конфигурациях между соприкасающимися массивными фазами - расплавом и кристаллом - в модели ПДЗ в пространстве концентраций описывают частотами отрыва мономера роста $\beta N^{-1}$ от мономера роста $\alpha N^{-1}$, принадлежащими узлам подрешеток « $j »$ и « $k »$ соответственно кристаллической фазы в конфигурации, характеризуемой высотами $K_{i}$ и $K_{i+1} \omega_{-\alpha \beta}^{(j k)}\left(K_{i}, K_{i+1}\right)$, частотами присоединения мономера роста $\beta N^{-1}$ расплава к мономеру $\alpha N^{-1}$ твердой фазы в соответствующих конфигурациях $\omega_{+\alpha \beta}^{(j k)}\left(K_{i}, K_{i+1}\right)$.

Эти частоты обменов мономерами роста были использованы в работах [1-5] и имеют следующий вид:

$$
\left\{\begin{array}{c}
v \exp \left[-\frac{\varepsilon_{\alpha \beta}^{11}+\sum_{\delta} \varepsilon_{\delta \beta}^{11} x_{\delta}^{(j)}}{T}\right]=\omega_{1 \alpha \beta}^{(j k)} \\
\text { при } K_{i}>0, K_{i+1}<0 ; \\
v \exp \left[-\frac{\varepsilon_{\alpha \beta}^{11}+2 \sum_{\delta} \varepsilon_{\delta \beta}^{11} x_{\delta}^{(j)}}{T}=\omega_{2 \alpha \beta}^{(j k)}\right] \\
\text { при } K_{i}>0, K_{i+1} \geq 0, \text { либо } K_{i} \leq 0, K_{i+1}<0 ; \\
v \exp \left[-\frac{\varepsilon_{\alpha \beta}^{11}+3 \sum_{\delta} \varepsilon_{\delta \beta}^{11} x_{\delta}^{(j)}}{T}\right]=\omega_{3 \alpha \beta}^{(j k)} \\
\text { при } K_{i} \leq 0, K_{i+1} \geq 0 .
\end{array}\right.
$$




$$
\begin{gathered}
\omega_{+\alpha \beta}^{(j k)}\left(K_{i}-N^{-1}, K_{i+1}\right)=\omega_{+1 \alpha \beta}^{(j k)}= \\
=v_{+} \exp \left[-\frac{\varepsilon_{\alpha \beta}^{12}+\sum_{\delta} \varepsilon_{\delta \beta}^{12} x_{\delta}^{(j)}}{T}\right] \\
\text { при } K_{i}-N^{-1}<0, K_{i+1}<0, \\
\omega_{+\beta \gamma}^{(j k)}\left(K_{i}, K_{i+1}\right)=\omega_{+1 \beta \gamma}^{(j k)}=v_{+} \exp \left[-\frac{\varepsilon_{\beta \gamma}^{12}+\sum_{\delta} \varepsilon_{\delta \gamma}^{12} x_{\delta}^{(j)}}{T}\right] \\
\text { при } K_{i}<0, K_{i+1} \leq 0 .
\end{gathered}
$$

В выражениях (3) и (4) постоянная Больцмана принята за единицу, $v_{+}$и $v$ есть частотные факторы, характеризующие степень колебаний около положений равновесий мономеров роста в двухкомпонентных металлических расплавах и в двухкомпонентных кристаллических фазах соответственно, величины $\varepsilon_{\alpha \beta}^{11}$ и $\varepsilon_{\alpha \beta}^{12}$ и им подобные означают взятые с обратным знаком энергии связи двух ближайших мономеров роста $\alpha N^{-1}$ и $\beta N^{-1}$, находящихся в кристалле, а также аналогичных мономеров роста кристалла и расплава. Индексы вверху 1 и 2 относятся к кристаллу и расплаву соответственно. Индексы, отмечающие номера подрешеток в кристаллической фазе при этом опущены. Функции $x_{\delta}^{(j)}$ определены равенствами (1) и (2).

В работах [1-4] было отмечено, что формулы (3) и (4) для частот присоединения мономеров роста из двухкомпонентного расплава к мономерам роста кристаллической фазы были справедливы при условии, что по крайней мере, один из мономеров роста находится в жидкой фазе (расплаве), типы же мономеров роста, взаимодействующих в первом приближении, при этом особой роли не играют. Эти обстоятельства выражаются в виде следующих равенств:

$$
\varepsilon_{A A}^{12}=\varepsilon_{A B}^{12}=\varepsilon_{A A}^{22}=\varepsilon_{A B}^{22}=\varepsilon_{B B}^{12}=\varepsilon_{B A}^{12}=\varepsilon_{B B}^{22}=\varepsilon_{B A}^{22}
$$

Из равенств (5) следует, что частоты присоединения, характеризующие переходы мономеров роста из расплава в двухкомпонентную кристаллическую фазу, не зависят от конкретной конфигурации ПДЗ, где происходят подобные обмены, но зависят от энергии связи ближайших односортных мономеров роста, находящихся в разных агрегатных состояниях и от температуры кристаллизующегося двухкомпонентного расплава. Если ввести параметр $q_{2}=\exp \left[-\frac{2 \varepsilon_{A A}^{12}}{T}\right]$, где $\varepsilon_{A A}^{12}-$ взятая с обратным знаком энергия взаимодействия односортных мономеров роста $A-A$, находящихся в разных агрегатных состояниях, то в силу равенств (5) можно получить для частот присоединения мономеров роста следующие равенства:

$$
\omega_{+a b}^{(j k)}\left(K_{i}-N^{-1}, K_{i+1}\right)=\omega_{+\beta \gamma}^{(j k)}\left(K_{i}, K_{i+1}\right)=v_{+} q_{2}
$$

при $K_{i}-N^{-1}<0, K_{i}<0, K_{i+1} \leq 0$

$$
\omega_{+\beta \gamma}^{(k, j)}\left(K_{i}, K_{i+1}\right)=\omega_{+1 \beta \gamma}^{(k, j)}=v_{+} q_{2} \text {. }
$$

Если ввести новую величину $q_{1}=\exp \left[-\frac{2 \varepsilon_{A A}^{11}}{T}\right]=$ $=\exp \left[-\frac{2 \varepsilon_{B B}^{11}}{T}\right]$, связанную с энергиями связи двух ближайших мономеров роста одного сорта в кристалле, то, используя условия (5) и равенства $\varepsilon_{A A}^{12}=\varepsilon_{A B}^{12}=n \varepsilon_{A A}^{11}=n \varepsilon_{B B}^{11}$, где $n$ обычно меньше единицы $\left(n=\frac{1}{2} ; \frac{1}{3}\right)$, то получаем равенства:

то есть

$$
q_{1}^{n}=\exp \left[-\frac{2 \varepsilon_{A B}^{12}}{T}\right]=\exp \left[-\frac{2 \varepsilon_{B A}^{12}}{T}\right],
$$

$$
q_{2}=q_{1}^{2 n}
$$

При $n=\frac{1}{2}$, имеем $q_{1}=q_{2}$. Эти результаты будут использованы при исследовании кристаллизации двухкомпонентных 50 \% металлических расплавов в диффузионно-релаксационном режиме. Связь энергий связи в приближении ближайших соседей между разносортными и односортными мономерами роста, находящихся в кристаллической фазе, есть $\varepsilon_{A B}^{11}=m \varepsilon_{A A}^{11}$, где $m>1$.

Физически модель конечной протяженности переходной двухфазной зоны в пространстве концентраций мономеров роста обусловлена механизмом спонтанных флуктуаций концентраций мономеров роста кристаллического состояния с ограниченным спектром изменения величин этих концентраций. Если ввести величину $c_{i}\left(t_{i}\right)=X_{i i}$, которая определяет величину концентрации мономеров роста после флуктуации в $i$-ом монослое переходной зоны в момент времени $t_{i}$, то концентрация мономеров роста кристаллической двухкомпонентной фазы равна ограниченной величине

$$
X_{i+1 i+1} \leq X_{i i} \leq X_{i-1 i-1},
$$

где $X_{i-1 i-1}=c_{i-1}\left(t_{i-1}\right) ; X_{i+1 i+1}=c_{i+1}\left(t_{i+1}\right)$ - соответствующие аналогичные концентрации в слоях $(i-1)$ в момент времени $t_{i-1}$ и $(i+1)$ в момент времени $t_{i+1}$, причем $t_{i-1}=t_{i}-\tau, t_{i+1}=t_{i}+\tau(\tau-$ период одной спонтанной флуктуации) (см. [5]). Тогда при законах распределения подобных флуктуаций можно ввести понятие величины средней концентрации $\bar{X}_{i i}$ мономеров роста твердого состояния в мо- 
нослое $i\left(i=1,2, \ldots, n_{0}\right)$ в момент времени $t_{i}$. Для хорошо развитых переходных двухфазных зон, характеризуемых большим числом монослоев $n_{0}$, теория спонтанных флуктуаций с ограниченным спектром изменения концентраций мономеров роста твердого состояния дает следующее выражение: $\bar{X}_{i i}=\left(\frac{1}{2}\right)^{i}, i\left(i=1,2, \ldots, n_{0}\right)$. При этом протяженность самой ПДЗ (число ее монослоев) отвечает условию $n_{0}<1+1.4 \ln N$.

В модели ПДЗ, связанной с механизмом спонтанных флуктуаций концентраций мономеров роста кристаллического состояния с ограниченным спектром изменения этих концентраций, размер $i$-го слоя в $t_{i}$ - момент дискретного времени, состоящего лишь из кристаллической двухкомпонентной фазы, очевидно равен $N \bar{X}_{i i} d$, где $d$-линейный размер мономера роста, $t_{i}=t_{0}+i \tau,\left(i=1,2, \ldots, n_{0}\right), t_{0}-$ начальный момент упорядоченного действия вышеупомянутого флуктуационного механизма. Очевидно время релаксации (установления упорядоченности двухкомпонентной кристаллической фазы), в рассматриваемой модели ПДЗ в каждом $i$-монослое $\left(i=1,2, \ldots, n_{0}\right)$ можно определить соотношением $\tau=N \bar{X}_{i i} / v$. Общая протяженность неоднородности по составу мономеров роста двухкомпонентной кристаллической фазы (сумма всех заштрихованных столбиков по высоте на рис. 1) равна

$$
L=N d \sum_{i=1}^{n_{0}} \bar{X}_{i i}
$$

Полное время релаксации, связанное с возможными перемещениями всех мономеров роста кристаллической двухкомпонентной фазы во всех $n_{0}$ монослоях сечения ПДЗ плоскостью $Z=0$, очевидно равно

$$
\tau_{p}=\sum_{i=1}^{n_{0}} \tau_{p i}=\frac{N}{v} \sum_{i=1}^{n_{0}} \bar{X}_{i i}=\frac{N}{v}\left[1-(1 / 2)^{n_{0}}\right],
$$

где $\sum_{i=1}^{n_{0}} \bar{X}_{i i}=1-(1 / 2)^{n_{0}}-$ конечная сумма членов убывающей геометрической прогрессии со знаменателем $q=1 / 2$. С другой стороны, если ввести коэффициент диффузии $D$, связанный с перемещением мономеров роста кристаллической фазы во всех $n_{0}$ монослоях ПДЗ, то из известного диффузионного соотношения $L^{2}=D \tau_{p}$ следует выражение для времени релаксации:

$$
\begin{aligned}
\tau_{p} & =\sum_{i=1}^{n_{0}} \tau_{p i}=\frac{N}{v}\left[1-(1 / 2)^{n_{0}}\right]=L^{2} / D, \\
\text { где }(N d)^{2} & =\left(\sum_{i=1}^{n_{0}} \bar{X}_{i i}\right)^{2}=\frac{D N}{v} \sum_{i=1}^{n_{0}} \bar{X}_{i i} .
\end{aligned}
$$

Таким образом, получаем выражение для времени релаксации во всей кристаллической двухкомпонентной фазе в модели ПДЗ:

$$
\tau_{p}=\frac{D}{(v d)^{2}}
$$

В таком случае усредненное время релаксации для взятого отдельно $i$-го монослоя ПДЗ можно записать в виде $\tau_{p i}=\tau_{p} / n_{0}$. Например, если считать $n_{0}=10 ; N=10^{5} ; v=10^{13}$ Гц, то оценка времени релаксации, даваемая флуктуационной теорией есть $\tau_{p i}=(N / v) \bar{X}_{i i}=10^{-11}$ сек. Еслиположить коэффициент диффузии в металлических двухкомпонентных сплавах в кристалле $D=10^{-6} \mathrm{M}^{2} / \mathrm{c},(v d)=10^{2} \mathrm{M} / \mathrm{c}$, то время релаксации в диффузионно-релаксационном режиме окажется равным $\tau_{p i}=\tau_{p} / n_{0}$, то есть времена релаксации в среднем даваемые флуктуационной теорией (см. [5]) и диффузионно-релаксационным режимом кристаллизации 50\% двухкомпонентных расплавов практически совпадают.

\section{УРАВНЕНИЯ ЭВОЛЮЦИИ ПЕРЕХОДНОЙ ДВУХФАЗНОЙ ЗОНЫ ПРИ \\ ДИФФУЗИОННО-РЕЛАКСАЦИОННОМ \\ РЕЖИМЕ КРИСТАЛЛИЗАЦИИ ДВУХКОМПОНЕНТНЫХ \\ 50 \% МЕТАЛЛИЧЕСКИХ РАСПЛАВОВ}

При переходе от кинетического режима кристаллизации двухкомпонентных 50 \% металлических расплавов, описанного в работах [1-8], к диффузионнорелаксационному режиму подобных расплавов изменение состояния переходной двухфазной зоны (ПДЗ) должно описываться структурными функциями, зависящими от времени, с учетом наличия времен релаксации $\tau_{p i}\left(i=1,2, \ldots, n_{0}\right)$. В соответствии со «ступенчатой» формой раздела двух соприкасающихся двухкомпонентных массивных фаз (расплава и кристалла) и при наличии времен релаксации дифференциально-разностные кинетические уравнения, описывающие эволюцию ПДЗ во времени и при учете условий $K_{i} \leq 0, K_{i+1} \leq 0$, имеют следующий вид:

$$
\begin{gathered}
\frac{d X_{\alpha \beta}^{(j k)}\left(K_{i}, t\right)}{d t}= \\
=\sum_{K_{i+1}=0}^{\left.K_{i+1}-\bar{c}_{i-1}\right)<0} \omega_{+\alpha \beta}^{(j k)}\left(K_{i}-N^{-1}, K_{i+1}\right) X_{\alpha}^{(j)}\left(K_{i}-N^{-1}, K_{i+1}, t\right)+ \\
+\sum_{\gamma} \sum_{\left.\bar{c}_{i+1}-\bar{c}_{i-1}\right)<0}^{K_{i+1}=0} \omega_{-\beta \gamma}^{(k j)}\left(K_{i}+N^{-1}, K_{i+1}\right) X_{\alpha \beta \gamma}^{(j k j)}\left(K_{i}+N^{-1}, K_{i+1}, t\right)- \\
-\sum_{\gamma} \sum_{K_{i+1}=0}^{\left(\bar{c}_{i+1}-\bar{c}_{i-1}\right)<0} \omega_{+\beta \gamma}^{(k j)}\left(K_{i}, K_{i+1}\right) X_{\alpha \beta}^{(j k)}\left(K_{i}, K_{i+1}, t\right)- \\
-\sum_{K_{i+1}=0}^{\left(\bar{c}_{i+1}-\bar{c}_{i-1}\right)<0} \omega_{-\alpha \beta}^{(j k)}\left(K_{i}, K_{i+1}\right) X_{\alpha \beta}^{(j k)}\left(K_{i}, K_{i+1}, t\right)=-\frac{X_{\alpha \beta}^{(j k)}\left(K_{i}, t\right)}{\tau_{p i}}
\end{gathered}
$$


где $\alpha, \beta, \gamma=A, B ; j, k=1,2$

Знак минус в правой части дифференциально-разностного уравнения (8) следует из физического требования на решения дифференциального уравнения

$$
\frac{d X_{\alpha \beta}^{(j k)}\left(K_{i}, t\right)}{d t}=\frac{d X_{\alpha \beta}^{(j k)}\left(K_{i}, t\right)}{\tau_{p i}}
$$

то есть на функцию

$$
X_{\alpha \beta}^{(j k)}\left(K_{i}, t\right)=X_{\alpha \beta}^{(j k)}\left(K_{i}, 0\right) e^{-\left(\frac{t}{\tau_{p i}}\right)}=Z_{\alpha \beta}^{(j k)}\left(K_{i}\right) e^{-\left(\frac{t}{\tau_{p i}}\right)},
$$

а именно $\lim _{t \rightarrow \infty} X_{\alpha \beta}^{(j k)}\left(K_{i}, t\right)=0$, что означает исчезновение переходной двухфазной зоны при достаточно большом интервале $t$, а, следовательно, и всех ее характеристик (процесс кристаллизации прекращается). При введении новых функций:

$$
\begin{aligned}
X_{\alpha}^{(j)}\left(K_{i}-N^{-1}, K_{i+1}, 0\right) & =Z_{\alpha}^{(j)}\left(K_{i}-N^{-1}, K_{i+1}\right) ; \\
X_{\alpha \beta}^{(j k)}\left(K_{i}, K_{i+1}, 0\right) & =Z_{\alpha \beta}^{(j k)}\left(K_{i}, K_{i+1}\right) ; \\
X_{\alpha \beta \gamma \gamma}^{(j k j)}\left(K_{i}+N^{-1}, K_{i+1}, 0\right) & =Z_{\alpha \beta \gamma}^{(j k j)}\left(K_{i}+N^{-1}, K_{i+1}\right) ;
\end{aligned}
$$

описывающих начало кристаллизации ПДЗ в момент времени $t=0$, уравнение (8) переходит в следующее:

$$
\begin{gathered}
\sum_{K_{i+1}=0}^{\left(\bar{c}_{i+1}-\bar{c}_{i-1}\right)<0} \omega_{+\alpha \beta}^{(j k)}\left(K_{i}-N^{-1}, K_{i+1}\right) Z_{\alpha}^{(j)}\left(K_{i}-N^{-1}, K_{i+1}\right)+ \\
+\sum_{\gamma} \sum_{K_{i+1}=0}^{\left(\bar{c}_{i+1}=\bar{c}_{i-1}\right)<0} \omega_{-\beta \gamma}^{(k j)}\left(K_{i}+N^{-1}, K_{i+1}\right) Z_{\alpha \beta \gamma}^{(j k j)}\left(K_{i}+N^{-1}, K_{i+1}\right)- \\
-\sum_{\gamma} \sum_{K_{i+1}=0}^{\left(\bar{c}_{i+1}-\bar{c}_{i-1}\right)<0} \omega_{+\beta \gamma}^{(k j)}\left(K_{i}, K_{i+1}\right) Z_{\alpha \beta}^{(j k)}\left(K_{i}, K_{i+1}\right)- \\
-\sum_{K_{i+1}=0}^{\left(\bar{c}_{i+1}-\bar{c}_{i-1}\right)<0} \omega_{-\alpha \beta}^{(j k)}\left(K_{i}, K_{i+1}\right) Z_{\alpha \beta}^{(j k)}\left(K_{i}, K_{i+1}\right)=-\frac{Z_{\alpha \beta}^{(j k)}\left(K_{i}\right)}{\tau_{p i}} .
\end{gathered}
$$

По аналогии с работами [1-4] все фигурирующие в уравнении функции $Z_{\alpha \beta}^{(j k)}\left(K_{i}\right)$, $Z_{\alpha \beta}^{(j k)}\left(K_{i}, K_{i+1}\right), Z_{\alpha \beta \gamma}^{(j k)}\left(K_{i}+N^{-1}, K_{i+1}\right)$ и им подобные отвечают условиям сверток по величинам $K_{i}, K_{i+1}$ и сортам мономеров роста $\alpha, \beta, \gamma=A, B$

$$
\begin{gathered}
\sum_{K_{i}, K_{i+1}} Z_{\alpha \beta}^{(j k)}\left(K_{i}, K_{i+1}\right)=\sum_{K_{i}} Z_{\alpha \beta}^{(j k)}\left(K_{i}\right)=Z_{\alpha \beta \beta}^{(j k)}, \\
\sum_{\alpha, \beta, \gamma} Z_{\alpha, \beta \gamma}^{(j k)}\left(K_{i}+N^{-1}, K_{i+1}\right)=Z\left(K_{i}+N^{-1}, K_{i+1}\right) .
\end{gathered}
$$

Одновременно имеют место условия нормировки для функций $Z_{\alpha \beta}^{(j k)}\left(K_{i}\right), Z_{\alpha \beta}^{(j k)}\left(K_{i}, K_{i+1}\right)$ :

$$
\sum_{\alpha \beta} \sum_{K_{i}} Z_{\alpha \beta}^{(j k)}\left(K_{i}\right)=1, \sum_{\alpha \beta} \sum_{K_{i}, K_{i+1}} Z_{\alpha \beta}^{(j k)}\left(K_{i}, K_{i+1}\right)=1
$$

и т. д. Сами функции $Z_{\alpha \beta}^{(j k)}\left(K_{i}\right)$ определены, аналогичны работам [1-4], в классе произведений

$$
Z_{\alpha \beta}^{(j k)}\left(K_{i}\right)=\left\{\begin{array}{l}
Z_{\alpha \beta}^{(j k)}(0) \lambda^{K_{i}} \text { при } K_{i} \geq 0, \\
Z_{\alpha \beta}^{(j k)}(0) \lambda^{\left|K_{i}\right|} \text { при } K_{i}<0,
\end{array}\right.
$$

где $\lambda$ заключена в пределах $0<\lambda \leq 1$ и носит название параметра «шероховатости» границы раздела фаз расплав-кристалл, $Z_{\alpha \beta}^{(j k)}(0)$ - функция, определяющая вероятность найти в модели ПДЗ столбик кристаллической фазы, оканчивающийся мономерами роста $\alpha N^{-1}, \beta N^{-1}$ принадлежащими подрешеткам « $j »$ и « $k$ » соответственно, у излома нулевой высоты. Корреляция соседних изломов в модели ПД3, представленной на рис. 1, по аналогии с работами [1-4] может быть записана в виде равенства для функций $Z_{\alpha \beta}^{(j k)}\left(K_{i}, K_{i+1}\right), Z_{\alpha \beta}^{(j k)}\left(K_{i}\right), Z_{\alpha \beta}^{(j k)}\left(K_{i+1}\right)$ :

$$
Z_{\alpha \beta}^{(j k)}\left(K_{i}, K_{i+1}\right)=\frac{Z_{\alpha \beta}^{(j k)}\left(K_{i}\right) Z_{\alpha \beta}^{(j k)}\left(K_{i+1}\right)}{Z_{\alpha \beta}^{(j k)}} .
$$

\section{С учетом вычисленных сумм}

$$
\begin{gathered}
\sum_{K_{i} \neq 0}^{\left(\bar{c}_{i+1}-\bar{c}_{i-1}\right)<0} \lambda^{\left|K_{i}-N^{-1}\right|}=\sum_{K_{i}=0}^{\left(\bar{c}_{i+1}-\bar{c}_{i_{-1}-1}\right)<0} \lambda^{\left|K_{i}-N^{-1}\right|}-\lambda^{N^{-1}}= \\
=\lambda^{2 N^{-1}}\left(\frac{1-\lambda^{\Delta_{i}}}{1-\lambda^{N^{-1}}}\right), \\
\sum_{K_{i} \neq 0}^{\left(\bar{c}_{i+1}-\bar{c}_{i-1}\right)<0} \lambda^{\left|K_{i}+N^{-1}\right|}=\sum_{K_{i+1}=0}^{\left(\bar{c}_{i_{i+1}}-\bar{c}_{\bar{c}_{i-1}}\right)<0} \lambda^{\left|K_{i}+N^{-1}\right|}-\lambda^{N^{-1}}=\frac{1-\lambda^{\Delta_{i}}}{1-\lambda^{N^{-1}}}, \\
\sum_{K_{i} \neq 0}^{\left(\bar{c}_{i} \neq 0\right.} \lambda^{\left|K_{i}\right|}=\sum_{K_{i}=0}^{\left.\bar{c}_{i+1}-\bar{c}_{i_{1-1}}\right)<0} \lambda^{\left|K_{i}\right|}-1=\lambda^{N^{-1}}\left(\frac{1-\lambda^{\Delta_{i}}}{1-\lambda^{N^{-1}}}\right), \\
\Delta_{i}=\left(\bar{c}_{i-1}-\bar{c}_{i+1}\right)>0 .
\end{gathered}
$$

соотношений для энергий связи мономеров роста (5), после ввода параметров $R=\left(v_{+} / v\right) q_{2}$, $R_{1}=\left(v_{+} / v\right) q_{2}^{1 / 2}$ и коэффициентов $S_{p \alpha \beta}^{(j k)}=\omega_{p \alpha \beta}^{(j k)} / v$, где $p=(1,2,3)$, оказывается при $\Delta_{i} \approx N^{-1}<<1$, что

1) величины $\left(1-\lambda^{N^{-1}}\right) \approx\left(1-\lambda^{\Delta_{i}}\right) \approx\left(1-\lambda^{N^{-1}+\Delta_{i}}\right)$ - одного порядка малости;

2) из инвариантности функций $Z_{\alpha \beta}^{(j k)}(0)=\mathrm{inv}$ для случая кристаллизации 50 \% двухкомпонентных металлических расплавов для кристаллической кубической элементарной решетки стехиометрического состава следует инвариантность частот $\omega_{p \alpha \beta}^{(j k)}=\operatorname{inv}$ и коэффициентов $S_{p \alpha \beta}^{(j k)}=$ invпри одновременном преобразовании $A \leftrightarrow B, j \leftrightarrow k(j, k=1,2)$. В таком случае из кинетического уравнения (10), описывающего эволюцию переходной двухфазной зоны в диффузионно-релаксационном режиме, при соблюдении выражений (11), (12), (13) может быть получена следующая линейная относительно функций $Z_{\alpha \beta}^{(j k)}(0)$ при $\alpha=A, \beta=B, j=1, k=2$ система уравнений с шестью неизвестными переменными $Z_{A A}^{(12)}(0), Z_{A B}^{(12)}(0), Z_{B A}^{(12)}(0), Z_{B B}^{(12)}(0), \lambda(\eta)$ и $\eta$ - параметра дальнего порядка, входящего неявным образом в $Z_{\alpha \beta}^{(j k)}(0)[\eta]-$ функции, когда $K_{i} \leq 0, K_{i+1} \leq 0$ : 


$$
\left\{\begin{array}{l}
a_{1} Z_{A A}^{(12)}(0)+a_{5} Z_{A B}^{(12)}(0)=0 ; \\
a_{5} Z_{A A}^{(12)}(0)+a_{2} Z_{A B}^{(12)}(0)=0 ; \\
a_{3} Z_{B A}^{(12)}(0)+a_{5} Z_{B B}^{(12)}(0)=0 ; \\
a_{5} Z_{B A}^{(12)}(0)+a_{4} Z_{B B}^{(12)}(0)=0 ; \\
b_{1} Z_{A A}^{(12)}(0)+b_{3} Z_{B A}^{(12)}(0)-b_{5} Z_{B B}^{(12)}(0)=R \lambda^{N^{-1}} ; \\
-b_{6} Z_{A A}^{(12)}(0)+b_{2} Z_{A B}^{(12)}(0)+b_{4} Z_{B B}^{(12)}(0)=R \lambda^{N^{-1}} .
\end{array}\right.
$$

Здесь фигурируют следующие функциональные коэффициенты:

$$
\begin{aligned}
& a_{1}=R \lambda^{2 N^{-1}}+\left(\frac{1}{v \tau_{p i}}-2 R\right) \lambda^{N^{-1}}+S_{3 A A}^{(12)}\left(1-\lambda^{N^{-1}}\right) \\
& a_{2}=R \lambda^{2 N^{-1}}+\left(\frac{1}{v \tau_{p i}}-2 R\right) \lambda^{N^{-1}}+S_{3 A B}^{(12)}\left(1-\lambda^{N^{-1}}\right) ; \\
& a_{3}=R \lambda^{2 N^{-1}}+\left(\frac{1}{v \tau_{p i}}-2 R\right) \lambda^{N^{-1}}+S_{3 B A}^{(12)}\left(1-\lambda^{N^{-1}}\right) ; \\
& a_{4}=R \lambda^{2 N^{-1}}+\left(\frac{1}{v \tau_{p i}}-2 R\right) \lambda^{N^{-1}}+S_{3 B B}^{(12)}\left(1-\lambda^{N^{-1}}\right) ; \\
& a_{5}=R \lambda^{2 N^{-1}} ; \\
& b_{1}=2 R_{1}-\frac{1}{v \tau_{p i}}+\lambda^{N^{-1}} S_{2 A A}^{(12)}+S_{3 A A}^{(12)} ; \\
& b_{2}=2 R_{1}-\frac{1}{v \tau_{p i}}+S_{3 A B}^{(12)}-\lambda^{2 N^{-1}} S_{1 A B}^{(12)} ; \\
& b_{3}=2 R_{1}-\frac{1}{v \tau_{p i}}+S_{3 B A}^{(12)}-\lambda^{N^{-1}} S_{1 B A}^{(12)} ; \\
& b_{4}=2 R_{1}-\frac{1}{v \tau_{p i}}+\lambda^{N^{-1}} S_{2 B B}^{(12)}+S_{3 B B}^{(12)} ; \\
& b_{6}=\lambda^{N^{-1}}\left(S_{2 B B}^{(12)}+\lambda^{N^{-1}} S_{1 B B}^{(12)}\right) ; \\
& \left.N^{-1} S_{1 A A}^{(12)}\right) \\
& b_{5}
\end{aligned}
$$

С учетом соотношений (1) функциональные коэффициенты $S_{p \alpha \beta}^{(12)}(\eta), p=1,2,3 ; \alpha, \beta=A, B$ имеют следующий вид:

$$
\begin{aligned}
& S_{1 A A}^{(12)}(\eta)=q_{1}^{1+\frac{1}{2}(1+\eta)+\frac{m}{2}(1-\eta)} ; S_{2 A A}^{(12)}(\eta)=q_{1}^{1+(1+\eta)+m(1-\eta)} ; \\
& S_{3 A A}^{(12)}(\eta)=q_{1}^{1+\frac{3}{2}(1+\eta)+\frac{3 m}{2}(1-\eta)} ; S_{1 A B}^{(12)}(\eta)=q_{1}^{m+\frac{m}{2}(1+\eta)+\frac{1}{2}(1-\eta)} ; \\
& S_{2 A B}^{(12)}(\eta)=q_{1}^{m+m(1+\eta)+(1-\eta)} ; S_{3 A B}^{(12)}(\eta)=q_{1}^{m+\frac{3 m}{2}(1+\eta)+\frac{3}{2}(1-\eta)} ; \\
& S_{1 B A}^{(12)}(\eta)=q_{1}^{m+\frac{1}{2}(1+\eta)+\frac{m}{2}(1-\eta)} ; S_{2 B A}^{(12)}(\eta)=q_{1}^{m+(1+\eta)+m(1-\eta)} ; \\
& S_{3 B A}^{(12)}(\eta)=q_{1}^{m+\frac{3}{2}(1+\eta)+\frac{3 m}{2}(1-\eta)} ; S_{1 B B}^{(12)}(\eta)=q_{1}^{1+\frac{m}{2}(1+\eta)+\frac{1}{2}(1-\eta)} ; \\
& S_{2 B B}^{(12)}(\eta)=q_{1}^{1+m(1+\eta)+(1-\eta)} ; S_{3 B B}^{(12)}(\eta)=q_{1}^{1+\frac{3 m}{2}(1+\eta)+\frac{3}{2}(1-\eta)}
\end{aligned}
$$

При этом преобразование $A \leftrightarrow B$ во всех функциональных коэффициентах эквивалентно преобразованию $\eta=-\eta$. Имеют место равенства:

$$
S_{p A A}^{(12)}(-\eta)=S_{p B B}^{(12)}(\eta), S_{p A B}^{(12)}(-\eta)=S_{p A B}^{(12)}(\eta)
$$

при $p=1,2,3$

Система шести независимых уравнений (14) инвариантна относительно преобразования $A \leftrightarrow B$, то есть при замене $\eta=-\eta$ остается неизменной. При этом в силу свойств коэффициентов $S_{p \alpha \beta}^{(12)}(\eta)$ при преобразовании $\eta=-\eta$ первое уравнение переходит в четвертое уравнение и наоборот, второе уравнение переходит в третье уравнение и наоборот, пятое уравнение переходит в шестое уравнение и наоборот. Это свойство инвариантности системы должно сохраняться при любых значениях параметра дальнего порядка $\eta(t)$, включая $\eta(t)=0$, когда двухкомпонентная кристаллическая фаза полностью разупорядочена, так и $\eta(t)=1$, когда двухкомпонентная кристаллическая фаза с простой кубической ячейкой и стехиометрического состава полностью упорядочена. Кроме того, все функции $Z_{\alpha \beta}^{(12)}(0)$ и функциональные коэффициенты $S_{p \alpha \beta}^{(j k)}$ инвариантны $\left(Z_{\alpha \beta}^{(12)}(0)=i n v, S_{p \alpha \beta}^{(j k)}=i n v\right)$ относительно одновременных преобразований $A \leftrightarrow B, 1 \leftrightarrow 2$.

\section{КИНЕТИКА КРИСТАЛЛИЗАЦИИ ПЕРЕХОДНОЙ ДВУХФАЗНОЙ ЗОНЫ В ДИФФУЗИОННО-РЕЛАКСАЦИОННОМ РЕЖИМЕ ДЛЯ ДВУХКОМПОНЕНТНЫХ 50 \% МЕТАЛЛИЧЕСКИХ РАСПЛАВОВ}

В диффузионно-релаксационном режиме кристаллизации двухкомпонентных 50 \% металлических расплавов посредством введения функций $X_{\beta}^{(k)}\left(K_{i}, K_{i+1}, t\right), X_{\alpha \beta}^{(j k)}\left(K_{i}, K_{i+1}, t\right)$ и частот обмена мономеров роста между двухкомпонентным расплавом и кристаллом $\omega_{+\beta \gamma}^{(k j)}\left(K_{i}, K_{i+1}\right), \omega_{-\alpha \beta}^{(k j)}\left(K_{i}, K_{i+1}\right)$ среднюю скорость эволюции ПДЗ можно определить в виде:

$$
V(t)=\sum_{i=1}^{n_{0}} V_{i}(t)
$$

где

$$
\begin{gathered}
V_{i}(t)=\sum_{K_{i} K_{i+1}=0}^{\left(\bar{c}_{i+1}-\bar{c}_{i-1}\right)<0} \sum_{\beta \gamma} \omega_{+\beta \gamma}^{(k j)}\left(K_{i}, K_{i+1}\right) X_{\beta}^{(k)}\left(K_{i}, K_{i+1}, t\right)- \\
-\sum_{K_{i} K_{i+1}=0}^{\left(\bar{c}_{i+1}-\bar{c}_{i-1}\right)<0} \sum_{\alpha \beta} \omega_{-\alpha \beta}^{(j k)}\left(K_{i}, K_{i+1}\right) X_{\alpha \beta}^{(j k)}\left(K_{i}, K_{i+1}, t\right) .
\end{gathered}
$$

На языке функций $Z_{\beta}^{(k)}\left(K_{i}, K_{i+1}\right), Z_{\alpha \beta}^{(j k)}\left(K_{i}, K_{i+1}\right)$ выражение для $i$-ой компоненты средней скорости $V(t)$ имеет вид: 


$$
\begin{gathered}
V_{i}(t)=\left[\sum_{K_{i} K_{i+1}=0}^{\left(\bar{c}_{i+1}-\bar{c}_{i-1}\right)<0} \sum_{\beta \gamma} \omega_{+\beta \gamma}^{(k)}\left(K_{i}, K_{i+1}\right) Z_{\beta}^{(k)}\left(K_{i}, K_{i+1}\right)-\right. \\
\left.-\sum_{K_{i} K_{i+1}=0}^{\left(\bar{c}_{i+1}-\bar{c}_{i-1}\right)<0} \sum_{\alpha \beta} \omega_{-\alpha \beta}^{(j k)}\left(K_{i}, K_{i+1}\right) Z_{\alpha \beta}^{(j k)}\left(K_{i}, K_{i+1}\right)\right] e^{-\left(t / \tau_{p i}\right)} .
\end{gathered}
$$

С учетом равенства $\omega_{+\beta \gamma}^{(k j)}\left(K_{i}, K_{i+1}\right)=\omega_{1 \beta \gamma}^{(k j)}=v_{+} q_{2}$, а также сумм (13), выражений (11) и (12) первая четверная сумма в формуле (18) может быть представлена в виде:

$$
\begin{aligned}
& \sum_{K_{i} K_{i+1}=0}^{\left(\bar{c}_{i+1}-\bar{c}_{i-1}\right)<0} \sum_{\beta \gamma} \omega_{+\beta \gamma}^{(k j)}\left(K_{i}, K_{i+1}\right) Z_{\beta}^{(k)}\left(K_{i}, K_{i+1}\right)= \\
& =\frac{2 \nu_{+} q_{2}^{1 / 2}}{1-\lambda^{N^{-1}+\Delta_{i}}}\left[\left(1-\lambda^{N^{-1}}\right)+q_{2}^{1 / 2} \lambda^{N^{-1}}\left(1-\lambda^{\Delta_{i}}\right)\right] .
\end{aligned}
$$

Вторая четверная сумма в выражении (18) в том же приближении имеет следующий вид:

$$
\begin{gathered}
\sum_{K_{i} K_{i+1}=0}^{\left(\bar{c}_{i+1}-\bar{c}_{i_{i-1}}\right)<0} \sum_{\beta \gamma} \omega_{+\beta \gamma}^{(k j)}\left(K_{i}, K_{i+1}\right) Z_{\beta}^{(k)}\left(K_{i}, K_{i+1}\right)= \\
=\lambda^{N^{-1}}\left(\frac{1-\lambda^{\Delta_{i}}}{1-\lambda^{N^{-1}}}\right) \sum_{\alpha \beta} \omega_{2 \alpha \beta}^{(j k)} Z_{\alpha \beta}^{(j k)}(0)+\sum_{\alpha \beta} \omega_{3 \alpha \beta}^{(j k)} Z_{\alpha \beta}^{(j k)}(0) .
\end{gathered}
$$

После введения параметров $R=\left(v_{+} / v\right) q_{2}$, $R_{1}=\left(v_{+} / v\right) q_{2}^{1 / 2}$ коэффициентов $S_{2 \alpha \beta}^{(j k)}=\omega_{2 \alpha \beta}^{(j k)} / v$, $S_{3 \alpha \beta}^{(j k)}=\omega_{3 \alpha \beta}^{(j k)} / v$ выражение для приведенной $i$-ой компоненты средней скорости кристаллизации ПДз $\bar{V}_{i}=V_{i} / v$ может быть записано в виде:

$$
\begin{gathered}
\bar{V}_{i}=\frac{2}{1-\lambda^{N^{-1+\Delta_{i}}}}\left[R \lambda^{N^{-1}}\left(1-\lambda^{\Delta_{i}}\right)+R_{1}\left(1-\lambda^{N^{-1}}\right)\right]- \\
-\lambda^{N^{-1}}\left(\frac{1-\lambda^{\Delta_{i}}}{1-\lambda^{N^{-1}}}\right) \sum_{\alpha, \beta} S_{2 \alpha \beta}^{(j k)} Z_{\alpha \beta}^{(j k)}(0)-\sum_{\alpha, \beta} S_{3 \alpha \beta}^{(j k)} Z_{\alpha \beta}^{(j k)}(0) .
\end{gathered}
$$

Если ввести новую функцию

$F\left(R, R_{1}, \lambda\right)=\frac{2}{1-\lambda^{N^{-1+\Delta_{i}}}}\left[R \lambda^{N^{-1}}\left(1-\lambda^{\Delta_{i}}\right)+R_{1}\left(1-\lambda^{N^{-1}}\right)\right]$,

для разности концентраций мономеров роста твердого состояния в монослоях $(i-1)$ и $(i+1)$ принять минимальное значение, то есть $\Delta_{i}=2 N^{-1}$, то можно получить выражение для приведенной $i$-ой компоненты скорости кристаллизации ПДЗ в следующем виде:

$$
\begin{gathered}
\bar{V}_{i}=\frac{2\left[R_{1}+R \lambda^{N^{-1}}\left(1+\lambda^{N^{-1}}\right)\right]}{1+\lambda^{N^{-1}}+\lambda^{2 N^{-1}}}- \\
-\lambda^{N^{-1}}\left(1+\lambda^{N^{-1}}\right) \sum_{\alpha, \beta} S_{2 \alpha \beta}^{(j k)} Z_{\alpha \beta}^{(j k)}(0)-\sum_{\alpha, \beta} S_{3 \alpha \beta}^{(j k)} Z_{\alpha \beta}^{(j k)}(0)= \\
=F\left(R, R_{1}, \lambda\right)-\lambda^{N^{-1}}\left(1+\lambda^{N^{-1}}\right) \sum_{\alpha, \beta} S_{2 \alpha \beta}^{(j k)} Z_{\alpha \beta}^{(j k)}(0)- \\
-\sum_{\alpha, \beta} S_{3 \alpha \beta}^{(j k)} Z_{\alpha \beta}^{(j k)}(0) .
\end{gathered}
$$

Соответственно эволюция во времени приведенной скорости кристаллизации ПДЗ с учетом релаксационных процессов восстановления упорядоченной двухкомпонентной кристаллической фазы может быть представлена в виде:

$$
\begin{gathered}
\bar{V}_{i}(t)=\sum_{i=1}^{n_{0}} \bar{V}_{i}(t), \\
\bar{V}_{i}(t)=\left\{\frac{\left[R_{q}+R \lambda^{N^{-1}}\left(1+\lambda^{N^{-1}}\right)\right]}{1+\lambda^{N^{-1}}+\lambda^{2 N^{-1}}-}\right. \\
-\lambda^{N^{-1}}\left(1+\lambda^{N^{-1}}\right) \sum_{\alpha, \beta} S_{2 \alpha \beta}^{(j k)} Z_{\alpha \beta \beta}^{(j k)}(0)- \\
\left.-\sum_{\alpha, \beta} S_{3 \alpha \beta}^{(j k)} Z_{\alpha \beta}^{(j k)}(0)\right\} e^{-\left(t / \tau_{p i}\right)} .
\end{gathered}
$$

Здесь использованы формулы (17)-(21).

\section{НАУЧНАЯ И ПРАКТИЧЕСКАЯ ЗНАЧИМОСТЬ}

В представленной работе данные имеют общий характер и применимы для любых двухкомпонентных металлических кристаллов с простой кубической элементарной ячейкой и стехиометрического состава, растущих из двухкомпонентных 50 \% металлических расплавов в диффузионнорелаксационном режиме кристаллизации. Научная значимость подтверждается тем обстоятельством, что исследуемая модель микрокристаллизации двухкомпонентных $50 \%$ расплавов в модели ПДЗ наиболее близка к реально наблюдаемым на эксперименте процессам обмена микрочастицами между двумя соприкасающимися массивными фазами, находящимися в разных агрегатных состояниях. Представленная выше модель микрокристаллизации двухкомпонентных расплавов в диффузионно-релаксационном режиме с элементарной кубической решеткой близка по своей природе к процессам гетероэндотаксии металлов и полупроводников, когда на изначальной металлической подложке накладываются слои друг на друга разных компонент [14]. Последующие результаты исследования получения полностью разупорядоченной двухкомпонентной кристаллической фазы позволяют дать практические рекомендации по выращиванию соответствующих металлических кристаллов с совершенной внутренней упорядоченной структурой в диффузионно-релаксационном режиме кристаллизации. 


\section{ЗАКЛЮЧЕНИЕ}

В данной работе представлена новая модель микрокристаллизации в модели переходной двухфазной зоны в пространстве концентраций мономеров роста твердой двухкомпонентной фазы с простой кубической ячейкой и стехиометрического состава в диффузионно-релаксационном режиме. Определена в общем виде кинетика эволюции ПДЗ во времени через основные функциональные характеристики структуры переходной конечной протяженности области, отделяющей две массивные соприкасающиеся фазы - расплав и кристалл. Получена система шести неоднородных трансцендентных независимых уравнений, обладающих свойством инвариантности при преобразовании $\eta=-\eta$. В случае кристаллизации двухкомпонентных 50\% металлических расплавов с образованием кристаллической двухкомпонентной фазы с простой кубической решеткой это преобразование эквивалентно инвариантности всех функций и других параметров относительно переобозначений $A \leftrightarrow B$. Новая модель микрокристаллизации $50 \%$ двухкомпонентных металлических расплавов позволяет изучить процессы перехода от возникающей при определенных условиях термодинамики неупорядоченной кристаллической фазы к полностью упорядоченной фазе, а также выявить времена релаксации, способствующие этому процессу.

\section{КОНФЛИКТ ИНТЕРЕСОВ}

Авторы декларируют отсутствие явных и потенциальных конфликтов интересов, связанных с публикацией настоящей статьи.

\section{СПИСОК ЛИТЕРАТУРЫ}

1. Байков Ю. А., Петров Н. И. // Известия высших учебныхх заведений. Физика, 2014, т. 57, № 4, с. 35-43. URL: https://elibrary.ru/item.asp?id=21564600

2. Байков Ю. А., Петров Н. И. // Известия высших учебных заведений. Физика, 2014, т. 57, № 5, с. 32-44. URL: https://elibrary.ru/item.asp?id=21689205
3. Байков Ю. А., Петров Н. И. // Вестник МГОУ. Сер. «Физика-Математика», 2014, № 2, с. 63. URL: https://www.vestnik-mgou.ru/Articles/Doc/7435

4. Петров Н. И. Исследование прочессов разупорядочения кристаллов при их росте из двухкомпонентных металлических расплавов. Дис. ... канд. физ-мат наук. Национальный исследовательский технологический университет «МИСиС». Москва, 2017. 180 с. URL: http://misis.ru/files/6902/Petrov_AR.pdf

5. Саркисов П. Д., Байков Ю. А., Мешалкин В. П. Математическое моделирование кристаллизации однои двухкомпонентных металлических расплавов. М: Физматлит, 2003, 378 c. URL: https://istina.msu.ru/publications/book/101828661/

6. Sarkisov P. D., Baikov Yu. A., Meshalkin V. P. // Collection of Works. The Optimization of Composition, Structure and Properties of Metals, Oxides, Composites, Nano- and Amorphous Materials, Russia-Israel Conference, Moscow - Yekaterinburg, 2002. p. 172. URL: https://istina. msu.ru/publications/article/103469568/

7. Sarkisov P. D., Meshalkin V. P., Baikov Yu. A. // Collection of Works. The Optimization of Composition, Structure and Properties of Metals, Oxides, Composites, Nano- and Amorphous Materials, Russia-Israel Conference, Moscow - Yekaterinburg, 2002. p. 184. URL: https://istina. msu.ru/publications/article/103469593/

8. Саркисов П. Д., Байков Ю. А., Мешалкин В. П. // ДАН РФ, 2003, т. 390, № 6, с. 763. URL: https://elibrary. ru/item. asp?id $=17396040$

9. Байков Ю. А., Чистяков Ю. Д. // Известия АН СССР, сер. Металлье, 1990, № 4, с. 53.

10. Байков Ю. А., Чистяков Ю. Д. // Известия АН СССР, сер. Металль, 1991, № 3, с. 62.

11. Baikov Yu. A., Zelenev Yu. V., Haubenreisser W., Pfeiffer H. // Phys. Stat. Solidi (a), 1980, Bd. 61, № 2, p. 435.

12. Chistyakov Yu. D., Baikov Yu. A., Schneider H. G., Ruth V. // Crystal Research and Technology, 1985, vol. 20, № 8, 1985, p. 1007. URL: https://elibrary.ru/item. asp?id=31861841

13. Chistyakov Yu. D., Baikov Yu. A., Schneider H. G., Ruth V. // Crystal Research and Technology, 1985, vol. 20, № 9, 1985. p. 1149. URL: https://elibrary.ru/item. asp?id $=31831082$

14. Schneider H. G. Collection: Advances in Epitaxy and Endotaxy. Akademiai Kiado, Budapest, 1976, p. 23. 
UDC 669.017.3:536.421.4

DOI: $10.17308 / \mathrm{kcmf} .2019 .21 / 711$

Received 30.11.2018

Accepted 15.02.2019

\title{
THE MODEL OF MICROCRYSTALLIZATION OF 50 \% BINARY METALLIC MELTS IN THE DIFFUSIVE RELAXATION MODE
}

\author{
(C) 2019 Yu. A. Baikov ${ }^{1}$, N. I. Petrov*1, M. I. Timoshina ${ }^{2}$, E. V. Akimov ${ }^{2}$ \\ ${ }^{1}$ D. Mendeleev University of Chemical Technology of Russia \\ 9 Miusskaya sq., 125047 Moscow, Russian Federation \\ ${ }^{2}$ Moscow Technical University of Communications and Informatics \\ 8a, Aviamotornaya str., 111024 Moscow, Russian Federation
}

\begin{abstract}
Objective. This research investigates the model of microcrystallization of $50 \%$ binary metallic melts in the diffusive-relaxation procedure. The authors developed a scheme of a diphase transition region (DTR) defined in the concentration space of selected growth monomers.

Methods and methodology. The concentration of growth monomers belonged to two different aggregate states: the melt and the crystalline phase. A kinetic differential-difference equation describing the time evolution of the DTR structure was developed. The study used the model of discontinuous DTR configuration formed by the fluctuation mechanism of the above-mentioned concentrations of growth monomers of a limited spectrum. The DTR separates two massive adjoining phases - the binary melt and the crystal.

Results. Using approximate nearest neighbours the study defined the frequencies of the growth monomer exchange between the two adjoining massive phases, the binary melt and the crystal, as functions of their interaction energies and the temperature of the crystallizing system. This model corresponds to the real length interface of two contiguous phases, and is known as the Kossel-Stranski crystal.

Conclusion. The average crystallization rate of so-called diphase transition region (DTR) in the concentration space of growth monomers was recorded. The definition of diphase transition region kinetics took into account the principal peculiarities of the concentrations of spontaneous fluctuations of limited spectrum, i.e. its discontinuous form. Moreover, some problems associated with the orderdisorder processes in binary crystalline phases were considered.
\end{abstract}

Keywords: diphase transition region, growth monomers, a far-order parameter, the growth, the "roughness" parameter, the crystallization rate.

\section{CONFLICT OF INTEREST}

The authors declare the absence of obvious and potential conflicts of interest related to the publication of this article.

\section{REFERENCES}

1. Baikov Yu. A., Petrov N. I. Russian Physics Journal, 2014, vol. 57, no. 4, pp. 459-468. DOI: 10.1007/s11182014-0262-2

2. Baikov Yu. A., Petrov N. I. Russian Physics Journal, 2014, vol. 57, no. 5, pp. 598-614. DOI: 10.1007/s11182014-0282-y

3. Baikov Yu. A., Petrov N. I. Vestnik of $M G O U$, ser. "Physics and Mathematics", 2014, no. 2, p. 63. URL: https:// www.vestnik-mgou.ru/Articles/Doc/7435 (in Russ.)
4. Petrov N. I. The Crystal Disordering Study When Growing From the Binary Metallic Melts. National University of Science and Technology «MISiS» Dis. Cand. Phys. - Mat. Sci. Moscow, 2017, 180 p. URL: http://misis.ru/ files/6902/Petrov_AR.pdf (in Russ.)

5. Sarkisov P. D., Baikov Yu. A., Meshalkin V. P. The One- and Binary Metallic Melts Mathematical Modeling Crystallization. Moscow, Physmatlit Publ., 2003, 378 p. URL: https://istina.msu.ru/publications/book/101828661/ (in Russ.)

6. Sarkisov P. D., Baikov Yu. A., Meshalkin V. P. Collection of Works. "The Optimization of Composition, Structure and Properties of Metals, Oxides, Composites, Nanoand Amorphous Materials", Russia-Israel Conference, Moscow - Yekaterinburg, 2002, p. 172. URL: https://istina. msu.ru/publications/article/103469568/

$\checkmark$ Petrov Nikolai I., e-mail: nik petrov@mail.ru 
7. Sarkisov P. D., Meshalkin V. P., Baikov Yu. A. Collection of Works. "The Optimization of Composition, Structure and Properties of Metals, Oxides, Composites, Nanoand Amorphous Materials", Russia-Israel Conference, Moscow-Yekaterinburg, 2002, p. 184. URL: https://istina. msu.ru/publications/article/103469593/

8. Sarkisov P. D., Baikov Yu. A., Meshalkin V. P. Doklady Physics, 2003, vol. 48, no. 6, pp. 290-295. DOI: 10.1134/1.1591316

9. Baikov Yu. A., Chistyakov Yu. D. News of USSR Academy of Sciences, ser. Metals, 1990, no. 4, p. 53. (in Russ.)
10. Baikov Yu. A., Chistyakov Yu. D. News of USSR Academy of Sciences, ser. Metals, 1991, no. 3, p. 62. (in Russ.)

11. Baikov Yu. A., Zelenev Yu. V., Haubenreisser W., Pfeiffer H. Phys. Stat. Solidi (a), 1980, Bd. 61, no. 2, p. 435. DOI: 10.1002/pssa.2210610214

12. Chistyakov Yu. D., Baikov Yu. A., Schneider H. G., Ruth V. Crystal Research and Technology, 1985, vol. 20, no. 8, p. 1007. DOI: $10.1002 /$ crat. 2170200802

13. Chistyakov Yu. D., Baikov Yu. A., Schneider H. G., Ruth V. Crystal Research and Technology, 1985, vol. 20, no. 9, p. 1149. DOI: 10.1002/crat.2170200903

14. Schneider H. G. Collection: Advances in Epitaxy and Endotaxy. Akademiai Kiado, Budapest, 1976, p. 23.
Байков Юрий Алексеевич - Д. ф.-м. н., професcop, кафедра физики, Российский химико-технологический университет имени Д. И. Менделеева, Москва, Россия; e-mail: nik_petrov@mail.ru. ORCID iD 0000-0003-3890-2838.

Петров Николай Иванович - к. ф.-м. н., доцент, кафедра физики, Российский химико-технологический университет имени Д. И. Менделеева, Москва, Россия; e-mail: nik_petrov@mail.ru. ORCID iD 0000-0002-5360-0806.

Тимошина Маргарита Игоревна - к. т. н., доцент, кафедра физики, Московский технический университет связи и информатики, Москва, Россия; e-mail: ritatoo@rambler.ru. ORCID iD 00000001-8954-0473.

Акимов Евгений Владимирович - ассистент, кафедра физики, Московский технический университет связи и информатики, Москва, Россия; e-mail: akimovmtuci@mail.ru. ORCID iD 0000-0001-63393495.
Baikov Yury A. - Dr. Sci (Phys.-Math), Full Professor, Physics Department, D. Mendeleev University of Chemical Technology of Russia, Moscow, Russian Federation; e-mail: nik_petrov@mail.ru. ORCID iD 0000-0003-3890-2838.

Petrov Nikolai I. - Cand. Sci (Phys.-Math) Associate Professor, Physics Department, D. Mendeleev University of Chemical Technology of Russia, Moscow, Russian Federation; e-mail: nik_petrov@mail.ru. ORCID iD 0000-0002-5360-0806.

Timoshina Margorita I. - Cand. Sci (Tech.) Associate Professor, Physics Department, Moscow Technical University of Communications and Informatics, Moscow, Russian Federation; e-mail: ritatoo@rambler.ru. ORCID iD 0000-0001-8954-0473.

Akimov Evgeniy V. - Lecturer, Physics Department, Moscow Technical University of Communications and Informatics, Moscow, Russian Federation; e-mail: akimovmtuci@mail.ru. ORCID iD 0000-00016339-3495. 